\title{
Aptidão física relacionada à saúde de escolares com idade de 7 a 10 anos
}

\section{Physical fitness related to the health of 7-10 year-old students}

Ana Paula Pietro Nobre Montoro, Carina Raffes Leite', Janine Aryadine Espíndola1, Juliano Maestri Alexandre, Monalisa da Silva Reis ${ }^{1}$, Renata Capistrano ${ }^{1}$, Tailine Lisboa ${ }^{1}$, Thais Silva Beltrame ${ }^{1,2}$

${ }^{1}$ Laboratório de Distúrbio da Aprendizagem e Desenvolvimento (LADADE), Centro de Ciências da Saúde e do Esporte (CEFID), Universidade do Estado de Santa Catarina (UDESC) - Florianópolis (SC), Brasil.

${ }^{2}$ Programa de Pós-Graduação em Ciências do Movimento Humano, UDESC - Florianópolis (SC), Brasil.

DOI: http://dx.doi.org/10.7322/abcshs.v41i1.842

\begin{abstract}
RESUMO
Introdução: A aptidão física relacionada à saúde refere-se às características físicas e fisiológicas que definem os perfis de morbimortalidade. Objetivo: Avaliar a aptidão física relacionada à saúde de escolares entre 7 e 10 anos. Métodos: Participaram do estudo 93 crianças de 7 a 10 anos de ambos os sexos. O instrumento utilizado foi a bateria de testes e medidas do Projeto Esporte Brasil (PROESP-BR) contendo teste de flexibilidade, resistência muscular, resistência cardiorrespiratória e índice de massa corporal (IMC), "peso/ altura2". Resultados: Percebe-se que os escolares apresentaram níveis desejados de aptidão física nos testes de flexibilidade e IMC; no entanto, nos testes de resistência muscular e cardiorrespiratória, os escolares obtiveram níveis indesejados, considerando risco à saúde. Conclusão: Os resultados observados não apresentam níveis satisfatórios de aptidão física relacionada à saúde em resistências muscular e cardiorrespiratória, revelando um risco de desenvolvimento de doenças crônicas não transmissíveis aos escolares.
\end{abstract}

Palavras-chave: aptidão física; promoção da saúde; criança; avaliação em saúde; atividade motora.

\section{ABSTRACT}

Introduction: The physical fitness related to health refers to the physical and physiological characteristics that define the morbidity and mortality profiles. Objective: To a Assess the physical fitness related to health of 7-10 year-old students. Methods: The study included 93 7-10 year-old students of both sexes. The instrument used was the battery of tests and measures of Projeto Esporte Brasil (PROESP-BR), including (flexibility test, muscular endurance, cardiorespiratory endurance and body mass index (BMI), "weight/height2". Results: It was observed that the students presented desired levels of physical fitness in the tests (flexibility and BMI), but in muscular endurance and cardiopulmonary tests, they got unwanted levels considering health risk. Conclusion: Our results do not show satisfactory levels of physical fitness related to health in muscular and cardiorespiratory endurance, revealing a risk of developing non transmissible chronic diseases to students.

Keywords: physical fitness; health promotion; child; health evaluation; motor activity. 


\section{INTRODUÇÃO}

A aptidão física relacionada à saúde refere-se às características físicas e fisiológicas que definem os perfis de morbimortalidade e os fatores associados às doenças crônicas, apresentando associação com prevalência de sedentarismo elevado em crianças e adolescentes ${ }^{1,2}$.

Por essa razão, o aproveitamento apropriado dos componentes da aptidão física relacionados à saúde (aptidão cardiorrespiratória, força/resistência muscular, flexibilidade e composição corporal), com a prática regular de atividade física na infância e na adolescência, traz diversos benefícios para a saúde ${ }^{2,3}$.

Em contrapartida, o baixo nível de atividade física habitual favorece o desenvolvimento de inúmeras disfunções crônicodegenerativas, como obesidade, dislipidemias, diabetes, doenças cardiovasculares, hipertensão, dentre tantas outras, em idade cada vez mais precoce ${ }^{4,5}$.

Diante desse quadro, a Organização Mundial da Saúde (OMS) incluiu a atividade física na Agenda Mundial de Saúde Pública, lançando a Estratégia Global de Alimentação, Atividade Física e Saúde. Entretanto, no que se refere à aptidão física no Brasil, na área do conhecimento científico, encontra-se escassa, por ter iniciado tardiamente ${ }^{6}$. Por outro lado, verifica-se na literatura que os poucos estudos que têm adotado abordagem em critérios relacionados à saúde passaram a ser desenvolvidos, em sua maioria, somente a partir da última década ${ }^{7-9}$.

Esse fato pode ser justificado pelas mudanças de paradigmas que vêm sendo observadas mundialmente nos hábitos de lazer e atividades de vida diária na população de crianças e adolescentes, devido ao interesse crescente por brincadeiras passivas e permanecer por diversas horas do dia diante de aparelhos de televisão e/ou microcomputadores ${ }^{10,11}$.

Nesse contexto, estudos epidemiológicos sugerem a prática regular de atividade física na infância e na adolescência, pelos diversos benefícios à saúde, seja pela sua influência direta sobre a prevenção de morbidades, seja pela sua influência no nível de atividade física na vida adulta.

Nessa linha, enquadrando-se nas metas de avaliação do Projeto Esporte Brasil (PROESP-BR), o objetivo deste estudo foi analisar, de acordo com sexo e idade, a aptidão física relacionada à saúde de escolares de 7 a 10 anos pertencentes a uma escola estadual de ensino situada no município de Florianópolis (SC).

\section{MÉTODO}

Realizou-se um estudo transversal, o qual envolveu 93 crianças de 7 a 10 anos, de ambos os sexos, pertencentes a uma escola estadual de ensino situada no município de Florianópolis (SC). Os participantes do estudo foram selecionados conforme a sua disponibilidade, sendo utilizada uma amostragem não probabilística.

O presente estudo faz parte de um projeto maior, intitulado "Dificuldades motoras, nível de aptidão física e percepção de competência em escolares da região de Florianópolis", aprovado pelo Comitê de Ética em Pesquisa em Seres Humanos da Universidade do Estado de Santa Catarina (CEPSH/UDESC), sob o protocolo $n^{\circ}$ 70/2011. Entretanto, nesta análise, foram explorados somente os dados referentes à aptidão física relacionada à saúde, utilizando o Manual de Aplicação de Medidas, Testes, Normas e Critérios de Avaliação do PROESP-BR ${ }^{12}$.

Os testes de aptidão física relacionada à saúde compreendem as análises de resistência cardiorrespiratória, resistência muscular localizada e flexibilidade e o estado nutricional. Sendo a resistência cardiorrespiratória verificada pelo teste de corrida de seis minutos; a flexibilidade, por meio do teste de sentar e alcançar; a resistência muscular localizada com a contagem do número de abdominais executados corretamente em um minuto (sit-up); e o estado nutricional, por meio da medida do peso e estatura para calcular o índice de massa corporal (IMC). Os resultados foram comparados aos critérios de referência apresentados no Manual do PROESP-BR para cada tarefa, os quais classificam as crianças em zona de risco à saúde ou zona saudável.

Quanto à coleta de dados, primeiramente foi realizado contato com o Setor Pedagógico e este repassado à Direção da Escola, a fim de receber a liberação para a aplicação dos testes. Assim, participaram do estudo apenas aqueles alunos pertencentes às turmas indicadas pela equipe pedagógica, bem como os que correspondessem aos critérios de inclusão da pesquisa, quais sejam: estar matriculado na instituição de ensino selecionada para o estudo; encontrar-se na faixa etária estabelecida (7 a 10 anos); intenção dos escolares para participarem de forma voluntária no estudo; apresentação da autorização dos pais e/ou responsáveis por meio da assinatura do Termo de Consentimento Livre e Esclarecido; ausência de problemas físicos nos membros inferiores ou superiores e de deficiências mentais que comprometessem a realização das avaliações, apontados previamente pela equipe pedagógica ou pelos pais.

Para a análise dos dados foi utilizado o Statistical Package for the Social Sciences (SPSS), versão 20.0, for Windows, sendo realizada uma estatística de análise de frequências (absoluta e relativa) e inferencial utilizando o teste $t$ de Student para amostra independente, com o objetivo de verificar se houve diferença entre os sexos nos testes de aptidão física relacionada à saúde, adotando o nível de significância $\mathrm{p}<0,05$.

\section{RESULTADOS}

Participaram do estudo 93 escolares, sendo 46 do sexo feminino e 47 do sexo masculino, com idade entre 7 e 10 anos, com média 9,01 $(1,09)$.

A Tabela 1 descreve as características dos participantes em média e desvio padrão das variáveis dos testes de aptidão física dividida por sexo, em que podemos observar que houve diferença significativa entre os sexos nas variáveis aptidão cardiorrespiratória $(\mathrm{p}=0,05)$ e flexibilidade $(\mathrm{p}=0,09)$. 
Tabela 1: Características gerais dos participantes

\begin{tabular}{|c|c|c|c|c|}
\hline Variáveis & $\begin{array}{c}\text { Meninos } \\
n=47 \\
\text { Média } \\
\text { DPP }\end{array}$ & $\begin{array}{c}\text { Meninas } \\
n=46 \\
\text { Média } \pm \text { DP }\end{array}$ & Total & Valor $p^{\star}$ \\
\hline Estatura $(\mathrm{cm})$ & $1,36 \pm 9,65$ & $1,36 \pm 17,93$ & $1,37 \pm 14,28$ & 0,85 \\
\hline Peso (kg) & $33,73 \pm 8,79$ & $35,53 \pm 8,95$ & $34,62 \pm 8,87$ & 0,33 \\
\hline IMC $\left(\mathrm{kg} / \mathrm{m}^{2}\right)$ & $17,65 \pm 3,16$ & $18,39 \pm 3,31$ & $18,02 \pm 3,24$ & 0,27 \\
\hline Aptidão cardiorrespiratória (m) & $727,07 \pm 110,10$ & $663,59 \pm 100,57$ & $696,04 \pm 109,70$ & $0,05^{*}$ \\
\hline Flexibilidade (cm) & $31,01 \pm 9,45$ & $36,31 \pm 9,48$ & $33,68 \pm 9,77$ & $0,09^{*}$ \\
\hline Abdominal (repetição por minuto) & $18,72 \pm 5,67$ & $17,28 \pm 7,20$ & $18,01 \pm 6,48$ & 0,28 \\
\hline
\end{tabular}

*Nível de significância do teste $t$ de Student para amostra independente $(p<0,05)$. DP: desvio padrão; IMC: índice de massa corporal.

A classificação dos resultados da aptidão física relacionada à saúde de crianças de 7 a 10 anos foi realizada conforme os critérios do Manual do PROESP-BR. Analisando os dados obtidos do IMC, verificouse que tanto as meninas quanto os meninos encontram-se, em sua maioria, na zona de saúde (64,5\% para ambos os sexos) (Tabela 2).

A Tabela 3 apresenta os resultados da flexibilidade obtidos pelo teste de sentar e alcançar. A análise de frequências mostra que a maioria dos escolares, tanto do sexo feminino quanto do masculino, encontra-se na zona de saúde, sendo 89,1 e 89,4\%, respectivamente.

No teste de resistência abdominal (Tabela 4), observa-se que mais da metade das meninas $(54,3 \%)$ e dos meninos $(68,1 \%)$ encontra-se na zona de risco à saúde.

A resistência cardiorrespiratória foi a aptidão física que apresentou um maior percentual de escolares na zona de risco. Conforme pode ser observado na Tabela 5, 91,5\% dos meninos e $87,0 \%$ das meninas encontram-se nessa condição.

\section{DISCUSSÃO}

As prevalências de sobrepeso e obesidade em crianças brasileiras têm aumentado nos últimos anos. Alguns fatores proporcionam o aumento de crianças obesas, como: alimentos com alto teor calórico, aumento do tempo gasto em aparelhos digitais, redução das atividades com gasto energético, influência dos pais na escolha alimentar e no nível de atividade física, levando cada vez mais ao sedentarismo ${ }^{13}$.

Em relação à prevalência de $64,5 \%$ de eutrofia, os dados obtidos neste estudo corroboram a literatura, em que $76,19 \%$ dos alunos foram classificados como eutróficos, 11,48\%, como obesos, 6,44\%, como sobrepeso, 2,8\%, como desnutridos, e 3,08\%, em risco de desnutrição ${ }^{13}$. Em estudo realizado por Ferreira et al. ${ }^{14}, 83 \%$ dos participantes foram classificados como normal, seguidos por $12,8 \%$, como sobrepeso. Estudo feito em SC, na cidade de Indaial, com 259 escolares com idade entre $8 \mathrm{e}$ 10 anos, também obteve resultados semelhantes aos do presente estudo, em relação ao sobrepeso, identificando $24,4 \%$ no sexo feminino e $17,7 \%$ no sexo masculino ${ }^{15}$. Outro estudo semelhante, de Rech et al. ${ }^{16}$, mostrou que 27,9\% dos escolares de ambos os sexos estavam acima do peso.

Para que a mudança do excesso de peso aconteça de forma concreta, é necessário conscientizar as autoridades de que essa epidemia, que se espalha mais a cada dia por todo o mundo e de forma abrupta, precisa ser trabalhada; assim, é necessário
Tabela 2: Resultado do índice de massa corporal

\begin{tabular}{|l|c|c|c|c|}
\hline IMC & $\begin{array}{c}\text { Meninas } \\
\mathbf{n}(\%)\end{array}$ & $\begin{array}{c}\text { Meninos } \\
\mathbf{n}(\%)\end{array}$ & Total & $\%$ \\
\hline Zona de saúde & $28(60,9)$ & $32(68,1)$ & 60 & 64,5 \\
\hline Zona de risco & $18(39,1)$ & $15(31,9)$ & 33 & 35,5 \\
\hline
\end{tabular}

IMC: índice de massa corporal.

Tabela 3: Resultados do teste de sentar e alcançar (flexibilidade)

\begin{tabular}{|l|c|c|c|c|}
\hline Flexibilidade & $\begin{array}{c}\text { Meninas } \\
\mathbf{n}(\%)\end{array}$ & $\begin{array}{c}\text { Meninos } \\
\mathbf{n}(\%)\end{array}$ & $\begin{array}{c}\text { Total } \\
\mathbf{n}\end{array}$ & $\%$ \\
\hline Zona de saúde & $41(89,1)$ & $42(89,4)$ & 83 & 89,2 \\
\hline Zona de risco & $6(10,9)$ & $5(10,6)$ & 10 & 10,8 \\
\hline
\end{tabular}

Tabela 4: Resultados do teste de resistência abdominal

\begin{tabular}{|l|c|c|c|c|}
\hline $\begin{array}{l}\text { Resistência } \\
\text { abdominal }\end{array}$ & $\begin{array}{c}\text { Meninas } \\
\mathbf{n}(\%)\end{array}$ & $\begin{array}{c}\text { Meninos } \\
\mathbf{n}(\%)\end{array}$ & $\begin{array}{c}\text { Total } \\
\mathbf{n}\end{array}$ & $\%$ \\
\hline Zona de saúde & $21(45,7)$ & $15(31,9)$ & 36 & 38,7 \\
\hline Zona de risco & $25(54,3)$ & $32(68,1)$ & 57 & 61,3 \\
\hline
\end{tabular}

Tabela 5: Resultados do teste de seis minutos (resistência cardiorrespiratória)

\begin{tabular}{|l|c|c|c|c|}
\hline $\begin{array}{l}\text { Aptidão } \\
\text { cardiorrespiratória }\end{array}$ & $\begin{array}{c}\text { Meninas } \\
\mathbf{n}(\%)\end{array}$ & $\begin{array}{c}\text { Meninos } \\
\mathbf{n}(\%)\end{array}$ & $\begin{array}{c}\text { Total } \\
\mathbf{n}\end{array}$ & $\%$ \\
\hline Zona de saúde & $6(13,0)$ & $4(8,5)$ & 10 & 10,8 \\
\hline Zona de risco & $40(87,0)$ & $43(91,5)$ & 83 & 89,2 \\
\hline
\end{tabular}

dispor de recursos como prevenções, desde a infância, visto que há probabilidade de uma criança obesa se tornar um adulto também obeso ${ }^{17}$.

Em relação aos resultados do teste de flexibilidade, somente cerca de $10 \%$ dos meninos e das meninas foram classificados na zona de risco, o que vai de encontro com a literatura. Em seu estudo, Fernandes et al. ${ }^{12}$ encontraram melhor desempenho no teste de flexibilidade nas meninas. O estudo de Ferreira et al. ${ }^{13}$ traz uma classificação em relação ao teste de flexibilidade, sendo caracterizado como "muito bom", "bom", "razoável", "fraco" e "muito fraco"; dessa forma, a maioria das crianças foi classificada na zona razoável (28,8\%), e outros $18,75 \%$ foram classificados como "muito fraco". 
A flexibilidade em bons níveis tem sido associada a menor incidência de lesões, principalmente na região dorsal e lombar, e está diretamente ligada à prevenção de doenças posturais ${ }^{18,19}$.

A avaliação da resistência abdominal também é de grande importância no contexto da aptidão física relacionada à saúde, visto que seu fraco desempenho pode causar problemas posturais e articulares e lesões musculoesqueléticas ${ }^{20,21}$. No presente estudo, a resistência abdominal apresentou valores preocupantes de $61,3 \%$ dos meninos e meninas classificados como zona de risco. $\mathrm{Na}$ literatura encontram-se diversos resultados, como o estudo de Mascarenhas et al. ${ }^{22}$, que assemelha-se ao presente estudo, com um de seus grupos alcançando 73,3\% dos escolares classificados como zona de risco. Porém, Luguetti et al. ${ }^{23}$ encontraram $36 \%$ de meninos e $40 \%$ de meninas com a pior classificação em seu teste.

Em relação ao teste de aptidão cardiorrespiratória, dos escolares avaliados, $87,0 \%$ dos meninos e $84,1 \%$ das meninas não atingiram os critérios de saúde. Resultados insatisfatórios também são encontrados em um estudo realizado em Santa Cruz do Sul (RS) com 1.666 escolares, em que 70,9\% dos meninos e 69,4\% das meninas avaliados não atingiram o nível esperado para a faixa etária ${ }^{24}$. Esses resultados são preocupantes, visto que, em um estudo realizado com crianças de 9 a 15 anos em Portugal, na Dinamarca e na Estônia, foi encontrada associação entre baixo nível de aptidão cardiorrespiratória e fatores de risco cardiovascular ${ }^{25,26}$. Além disso, a baixa aptidão aeróbia está associada a elevados índices de colesterol e triglicerídeos ${ }^{27,28}$, pressão arterial e sensibilidade à insulina desequilibradas ${ }^{27,29}$ e maior risco de obesidade ${ }^{30}$. Essa realidade está presente no estudo efetuado com escolares de Porto Velho (RO), uma vez que 53,25\% dos meninos e $63,76 \%$ das meninas encontram-se sedentários ou insuficientemente ativos ${ }^{25}$; consequentemente, essas crianças apresentam uma maior predisposição a adquirir algum tipo de comorbidade referente a estas relacionadas à baixa aptidão aeróbia.

Conclui-se que o presente estudo demonstrou bons resultados em relação ao IMC, indo ao encontro da literatura ${ }^{13-15}$.

Os indicadores de saúde utilizados da bateria de testes do PROESP apresentaram resultados diversos: com relação à flexibilidade, foi satisfatório, com 89,2\% dos escolares classificados como zona de saúde, apresentando diferença significativa entre os sexos. Entretanto, os resultados provenientes dos índices de resistência abdominal e aptidão cardiorrespiratória não foram satisfatórios, sendo que dos escolares avaliados, $54,3 \%$ para o masculino e $68,1 \%$ para o sexo feminino, respectivamente, foram classificados em zona de risco.

As informações produzidas no presente estudo contribuem para a literatura, demonstrando as condições dos escolares brasileiros e possivelmente alertando para ações que poderiam ser realizadas como forma de minimizar/melhorar essas condições apresentadas, visto que níveis abaixo do esperado contribuem para o desenvolvimento de possíveis doenças não transmissíveis.

\section{REFERÊNCIAS}

1. Andreasi V, Michelin E, Rinaldi AEM, Burin RC. Aptidão física associada às medidas antropométricas de escolares do ensino fundamental. J Pediatr. 2010;86(6):497-502.

http://dx.doi.org/10.1590/S0021-75572010000600009

2. Dumith SC, Azevedo Junior MR, Rombaldi AJ. Aptidão física relacionada à saúde de alunos do ensino fundamental do Município de Rio Grande, RS, Brasil. Rev Bras Med Esporte. 2008;14(5):454-9. http://dx.doi.org/10.1590/S1517-86922008000500011

3. Corbin CB, Pangrazi RP. Guidelines for appropriate physical activity for elementary school children 2003 Update. Reston: NASPE Publications; 2003.

4. Boreham C, Riddoch C. The physical activity, fitness and health of children. J Sports Sci. 2001:19(12):915-29 http://dx.doi.org/10.1080/026404101317108426

5. Santiago LM, Sá O, Carvalho IM, Rocha MG, Palmeiro L, Mesquita EP, et al. Hipercolesterolemia e factores de risco cardiovascular associados, em crianças e adolescentes. Rev Port Cardiol. 2002;21(3):301-13

6. Hallal PC, Dumith SC, Bastos JP, Reichert FF, Siqueira FV, Azevedo MR. Evolução da pesquisa epidemiológica em atividade física no Brasil: revisão sistemática. Rev Saúde Pública. 2007;41(3):453-60. http://dx.doi.org/10.1590/S0034-89102007000300018

7. Guedes DP, Guedes JERP. Aptidão física relacionado a saúde de crianças e adolescentes: avaliação referenciada por critério. Rev Bras Ativ Fis Saúde. 1995;1(2):27-38
8. Glaner MF. Nível de atividade física e aptidão física relacionada a saúde em rapazes rurais e urbanos. Rev Paul Educ Fis. 2002;16(1):76-85.

9. Serassuelo Junior $H$, Rodrigues AR, Cyrino ES, Ronque EV, Oliveira SRS, Simões AC. Aptidão física relacionada a saúde em escolares de baixo nível socioeconômico do município de Cambé/PR. Rev Educ Fís Uem. 2005;16(1):7-13. http://dx.doi.org/10.4025/reveducfisv16n1p5-11

10. Dietz WH, Gortmaker SL. Do we fatten our children at the television set? Obesity and television viewing in children and adolescents. Pediatrics. 1985;75(5):807-12.

11. Hancox RJ, Poulton R. Watching television is associated with childhood obesity: but is it clinically important? Int J Obes (London). 2006;30(1):171-5 http://dx.doi.org/10.1038/sj.ijo.0803071

12. Gaya A, Lemos A, Gaya A, Teixeira D, Pinheiro E, Moreira R Manual do Projeto Esporte Brasil - PROESP/BR. Brasília: Ministério do Esporte; 2012. Disponível em: http://www.proesp.ufrgs.br/ arquivos/Manual-PROESP-BR-2012.pdf. Acesso em: 03 fev. 2016.

13. Fernandes MM, PenhaDSG, BragaFA. Obesidade infantil em crianças da rede pública de ensino: prevalência e consequências para flexibilidade, força explosiva e velocidade. Rev Educ Fís. 2012;23(4):629-34. http://dx.doi.org/10.4025/reveducfis.v23.4.13991

14. Ferreira MVN, Saldanha MA, Silva RF, Araújo DME. A prevalência da obesidade e sobrepeso e níveis de flexibilidade em adolescentes de 14 a 18 anos. Fiep Bul. 2013;83. 
15. Bertin LR, Malkowski J, Zutter ICL, Ulbrich ZA. Estado nutricional, hábitos alimentares e conhecimentos de nutrição em escolares. Rev Paul Pediatr. 2010; 28(3):303-8. http://dx.doi.org/10.1590/S0103-05822010000300008

16. Rech RR, Halpern R, Costanzi CB, Bergmann MLA, Alli LR, Mattos AP, et.al. Prevalência de obesidade em escolares de 7 a 12 anos de uma cidade Serrana do RS, Brasil. Rev Bras Cineantropom Desempenho Hum. 2010;12(2):90-7. http://dx.doi.org/10.5007/1980-0037.2010v12n2p90

17. Abrantes MM, Lamounier JA, Colsimo EA. Prevalência de sobrepeso e obesidade nas regiões sudeste e nordeste. J Pediatr. 2002;78(4):335-40. http://dx.doi.org/10.1590/S0021-75572002000400014

18. Rodríguez PL, Santonja FM, Lopez-Miñarro PA, Sáinz de Baranda P, Yuste JL. Effect of physical education stretching programme on sitand-reach score in schoolchildren. Sci Sports. 2008;23(3-4):170-5.

19. Polachini LO, Fuzasaki L, Tamaso M, Tellini GG, Masieiro D. Estudo comparativo entre tres metodos de avaliação do encurtamento de musculatura posterior da coxa. Rev Bras Fisiot. 2005;9(2):187-93.

20. Knudson D. Issues in abdominal fitness: testing and technique. J Phys Educ Recr Dance. 1999;70(3):49-55. http://dx.doi.org/10.1080/07303084.1999.10605896

21. Silfies SP, Squillante D, Maurer P, Westcott S, Karduna AR. Trunk muscle recruitment patterns in specific chronic low back pain populations. Clin Biomech. 2005; 20(5):465-73. http://dx.doi.org/10.1016/j.clinbiomech.2005.01.007

22. Mascarenhas LPG, Ferreira AB, Lima VA, Grzelczak MT. Estudo comparativo da aptidão física entre crianças de escola pública e particular: uma visão regional. Cinergis. 2014;14(3):157-60.

23. Luguetti CN, Ré AHN, Böhme MTS. Indicadores de aptidão física de escolares da região centro-oeste da cidade de São Paulo. Rev Bras Cineantropom Desempenho Hum. 2010;12(5):331-7. http://dx.doi.org/10.5007/1980-0037.2010v12n5p331
24. Burgos MS, Reuter CP, Burgos LT, Pohl HH, Paiva DN, Reuter EM, et al. Aptidão cardiorrespiratória e fatores de risco cardiovasculares: um estudo com escolares de Santa Cruz do Sul, RS, Brasil. Rev Epidem Contr Infecção. 2013;3(4).

25. Farias ES, Salvador MRD. Antropometria, composição corporal e atividade física de escolares. Rev Bras Cineantropom Desempenho Hum. 2005;7(1):21-9.

26. Anderssen SA, Cooper AR, Riddoch C, Sardinha LB, Harro $\mathrm{M}$, Brage $\mathrm{S}$, et al. Low cardiorespiratory fitness is a strong predictor for clustering of cardiovascular disease risk factors in children independent of country, age and sex. Eur J Cardiovasc Prev Rehabil. 2007;14(4):526-31. http://dx.doi.org/10.1097/HJR.0b013e328011efc1

27. Eisenmann JC, Welk GJ, Ihmels M, Dollman J. Fatness, fitness, and cardiovascular disease risk factors in children and adolescents. Med Sci Sports Exerc. 2007; 39(8):1251-6. http://dx.doi.org/10.1249/MSS.0b013e318064c8b0

28. Perry AC, Okuyama T, Tanaka K, Signorile J, Kaplan TA, Wang X. A comparison of health and fitnessrelated variables in a small sample of children of Japanese descent on 2 continents. Arch Pediatr Adolesc Med. 2002;156(4):362-8 http://dx.doi.org/10.1001/archpedi.156.4.362

29. Carrel AL, Clark RR, Peterson SE, Nemeth BA, Sullivan J, Allen DB. Improvement of fitness, body composition, and insulin sensitivity in overweight children in a school-based exercise program: a randomized, controlled study. Arch Pediatr Adolesc Med. 2005;159(10):963-8

http://dx.doi.org/10.1001/archpedi.159.10.963

30. Kim J, Must A, Fitzmaurice GM, Gillman MW, Chomitz V, Kramer $E$, et al. Relationship of physical fitness to prevalence and incidence of overweight among schoolchildren. Obes Res. 2005;13(7):1246-54.

http://dx.doi.org/10.1038/oby.2005.148 